\title{
ANALYSIS OF THE TURBULENCE-RADIATION INTERACTION IN A METHANE-AIR DIFFUSION FLAME
}

\begin{abstract}
G. C. Fraga,
A. P. Petry,

and F. H. R. França

Universidade Federal do Rio Grande do Sul

Departamento de Engenharia Mecânica

Rua Sarmento Leite 425,

Porto Alegre, RS, Brasil guilhermecfraga@gmail.com
\end{abstract}

Received: April 14, 2018

Revised: May 21, 2018

Accepted: May 30, 2018

Keywords: turbulence-radiation interaction, diffusion flame, large eddy simulation, weighted-sum-of-gray-gases model, fire dynamics simulator

\section{NOMENCLATURE}

$a_{j} \quad$ temperature coefficient

$\mathrm{b}_{\mathrm{j}, \mathrm{k}} \quad$ polynomial coefficient, $\mathrm{K}^{-\mathrm{k}}$

$\mathrm{G}$ incident radiation, $\mathrm{W} / \mathrm{m}^{2}$

I radiation intensity, $\mathrm{W} / \mathrm{m}^{2}$

$\mathrm{I}_{\mathrm{b}} \quad$ blackbody radiation intensity, $\mathrm{W} / \mathrm{m}^{2}$

q radiative heat flux vector, $\mathrm{W} / \mathrm{m}^{2}$

$\mathrm{q}_{\mathrm{c}} \quad$ convective heat flux, $\mathrm{W} / \mathrm{m}^{2}$

$\mathrm{q}_{\mathrm{r}}$ radiative heat flux, $\mathrm{W} / \mathrm{m}^{2}$

$\mathrm{q}_{\mathrm{t}}$ total heat flux, $\mathrm{W} / \mathrm{m}^{2}$

$\mathrm{s} \quad$ path of radiation propagation, $\mathrm{m}$

$\mathrm{S}_{\mathrm{r}} \quad$ volumetric radiative heat source, $\mathrm{W} / \mathrm{m}^{3}$

$\mathrm{T}$ temperature, $\mathrm{K}$

$\mathrm{x}, \mathrm{y}, \mathrm{z}$ cartesian coordinates, $\mathrm{m}$

\section{Greek symbols}

$\eta \quad$ radiation wavenumber, $\mathrm{cm}^{-1}$

$\kappa \quad$ absorption coefficient, $\mathrm{m}^{-1}$

$\kappa_{\mathrm{p}}$ pressure-absorption coefficient, $\left(\mathrm{atm} \mathrm{m}^{-1}\right.$

$\Omega \quad$ solid angle

$\phi \quad$ arbitrary quantity

$\psi_{\phi} \quad$ Normalized difference for quantity $\phi$

\section{Subscripts}

j gray gas

$\eta \quad$ spectral dependence

\section{Superscripts}

nTRI solution neglecting turbulent fluctuations

TRI solution considering turbulent fluctuations

\section{INTRODUCTION}

When neglecting turbulent fluctuations, timeaveraged quantities such as the radiative heat flux and the radiative heat source may differ significantly from these same quantities computed using mean temperature and species concentration fields. This phenomenon is denominated turbulence-radiation interaction (TRI), and is caused by the highly nonlinear coupling between turbulence-promoted fluctuations of radiation intensity and fluctuations of temperature and medium composition (Modest, 2005; Coelho, 2007).

The importance of TRI for a number of different turbulent problems, especially those involving reacting 
flows, has been demonstrated experimentally, theoretically and numerically (Coelho, 2007). This paper focuses on turbulence-radiation interaction in a methane-air diffusion flame and, for this type of flame, previous studies report a wide range of values for the magnitude of TRI effects. For example, Jeng et al. (1984) used a stochastic model to determine the effects of turbulent fluctuations on the mean spectral radiation intensity to be of the order of 10 to $30 \%$. In another study, a joint probability density function (PDF) of velocity and medium composition was employed in the analysis of a methane-air diffusion flame stabilized downstream of a bluff-body (Mazumder and Modest, 1999), with findings pointing to an enhancement of the radiative emission from the flame due to TRI, which caused a decrease of the flame temperature by approximately $100 \mathrm{~K}$ and an increase of the wall radiative heat flux by about 45\%. Finally, in a second work adopting PDFs, Li and Modest (2002) simulated the combustion of methane in an axyssimetric configuration, and comparisons between calculations considering and neglecting turbulence-radiation interaction effects showed an increase of the radiative emission and of the radiative heat loss by more than $30 \%$ at the flame sheet, where turbulent fluctuations tend to be larger.

In this study, turbulence-radiation interaction is analyzed for methane-air diffusion flames using realistic transient fields generated from Large Eddy Simulation (LES). In order to evaluate the magnitude of TRI effects, time-averages of the wall heat fluxes and of the local volumetric radiative heat source, determined from the LES computations, are compared to results obtained from independent calculations initialized with mean temperature and species concentration fields. The radiative heat transfer is solved using the Weighted-Sum-of-GreyGases spectral model. All simulations are performed using the open-source, Fortran-based Fire Dynamics Simulator code (McGrattan et al., 2017a), implementing also some modified subroutines developed by the authors.

\section{PROBLEM STATEMENT}

Turbulence-radiation interaction is investigated in the context of a methane-air diffusion flame. Methane is injected through a central circular opening of diameter equal to $0.08 \mathrm{~m}$, and reacts with a co-flow of air, entering through an annular opening of $0.14 \mathrm{~m}$ of external diameter. The domain is a rectangular cavity, of total lateral dimensions equal to $0.4 \mathrm{~m}$ and length of $2 \mathrm{~m}$. The choice of a rectangular domain, rather than a cylindrical one, is due to a limitation of the code used for the simulations, which is not capable as of this moment to build geometry and numerical grids in a three-dimensional cylindrical coordinate system. Figure 1 depicts a schematic representation of the computational domain, highlighting the methane and air inlets.
All surfaces except the inlets of the methane and air streams and the domain's outlet (located at the surface opposite to the inlets) are walls with no-slip boundary condition, kept at a constant and uniform temperature of $20^{\circ} \mathrm{C}$ and assumed to behave as black surfaces for the radiative heat transfer calculations. The methane and air streams are also at $20^{\circ} \mathrm{C}$ at the domain entrance. In the cases considered in this paper, the inlet velocity of the methane stream varies between $1 \mathrm{~m} / \mathrm{s}$ and $3 \mathrm{~m} / \mathrm{s}$ as to provide a wide range of values for the Reynolds number (as discussed in the chapter of results), while the inlet velocity of the air stream is equal to $0.5 \mathrm{~m} / \mathrm{s}$ for all simulations. On both inlets, turbulence fluctuations are imposed using the Synthetic Eddy Method (Jarrin, 2008), considering $10 \%$ of turbulence intensity. At the domain's outlet, an open boundary condition to an outside environment maintained at $20^{\circ} \mathrm{C}$ and at atmospheric pressure is prescribed.
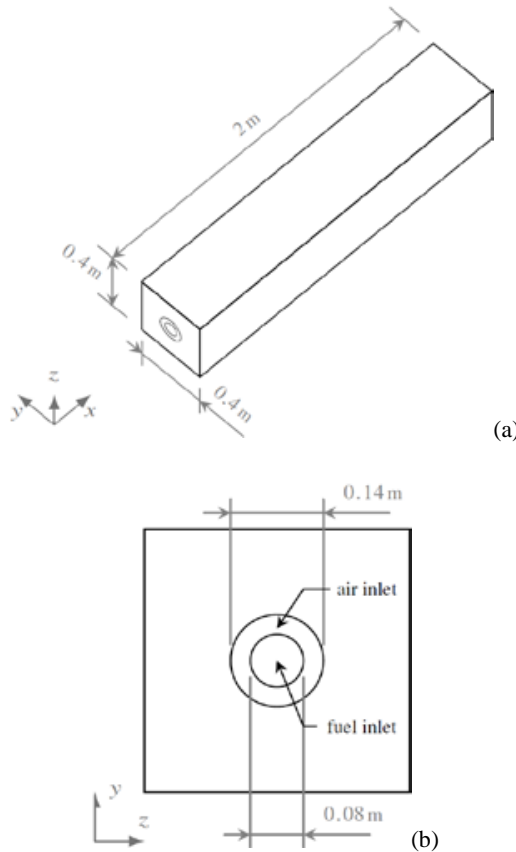

(a)

(b)

Figure 1. Geometry of the problem. (a) Threedimensional view; (b) front view identifying the fuel and air inlets.

\section{METHODOLOGY}

\section{Transport Equations, Turbulence Modeling and Combustion}

Transport equations for species mass fractions, momentum and energy are solved for a threedimensional, transient, non-isothermal, compressible flow in a Cartesian coordinate system. To simplify the governing equations and facilitate the numerical solution, the low Mach number approximation (Rehm and Baum, 1978) is introduced, allowing the separation of the total pressure in a background component, solved by a equation of state (for which it 
is assumed ideal gas behavior), and in a pressure fluctuation, which is determined through an additional Poisson equation (McGrattan et al., 2017b). A single step, mixing-controlling chemical reaction mechanism is adopted (Poinsot and Veynante, 2005). In order to lower the computational cost of the simulations, a lumped species approach is used, in which the species involved in the problem are combined into groups-namely, the fuel, the oxidant and the products-that transport and react together, thus reducing the number of transport equations that need to be solved (McGrattan et al., 2017b).

Large Eddy Simulation is employed to capture the transient fluctuations of all quantities of interest, through the application of a low-pass box filter whose width is determined by the grid cell size. For the closure of the LES transport equations, the dynamic Smagorinsky model is employed (Germano et al., 1991; Lilly, 1992) with constant turbulent Prandtl and Schmidt numbers, both equal to 0:5.

\section{Radiation Modeling}

The radiative heat transfer equation (RTE) is solved for the determination of the thermal radiation field. Assuming a non-scattering medium, as is the case for the problem studied in this paper, the RTE is given by (Howell et al., 2010; Modest, 2013):

$$
\frac{d I_{\eta}}{d s}=\kappa_{\eta} I_{b \eta}-\kappa_{\eta} I_{\eta}
$$

in which $\eta$ is the radiation wavenumber; $s$ is the coordinate along the path of radiation propagation; $\kappa_{\eta}$ is the spectral absorption coefficient of the medium; and $\mathrm{I}_{\eta}$ and $\mathrm{I}_{\mathrm{b \eta}}$ are the spectral radiation intensity and the blackbody spectral radiation intensity, respectively.

The RTE and the energy transport equation are coupled through the volumetric radiative heat source (which will be referred to in this paper as simply the radiative heat source), $S_{r}$, defined as the negative of the radiative heat flux divergence:

$$
\mathrm{S}_{\mathrm{r}}=-\nabla \cdot \mathbf{q}=\int_{0}^{\infty} \kappa_{\eta}\left(\mathrm{G}_{\eta}-4 \pi \mathrm{I}_{\mathrm{b \eta}}\right) \mathrm{d} \eta
$$

where $\mathbf{q}$ is the radiative heat flux vector and $G_{\eta}$ is the spectral incident radiation, given by the integration of the spectral intensity over all solid angles $\Omega$.

Spatial and spectral integrations of Eqs. (1) and (2) are required for the solution of the radiative heat transfer problem-i.e., integrations over all directions of radiation propagation and over all the radiation spectrum. For the former, the finite volume method (Raithby and Chui, 1990) is adopted. In this method, the unit sphere surrounding each point is divided in a finite number of solid angles, inside which the radiation intensity is assumed to be constant relative to direction; the RTE is then solved for each angle and the continuous integral over the unit sphere is approximated as a weighted summation over the intensity obtained for each discrete angle.

On the other hand, the Weighted-Sum-of-GrayGases (WSGG) model is used for the spectral integration of Eqs. (1) and (2). This is a relatively simple spectral model that has shown good agreement with results obtained by line-by-line integration (benchmark solutions) for a number of different situations (Dorigon et al., 2013; Cassol et al., 2014), without the requirement of elevated computational costs.

In the WSGG model, the spectrum of radiation is replaced by $\mathrm{N}_{\mathrm{j}}$ gray gases with uniform absorption coefficients and by transparent windows (with null absorption coefficients). The RTE for the jth gas may then be written as:

$$
\frac{d I_{j}}{d s}=\kappa_{j} a_{j} I_{b}-\kappa_{j} I_{j}
$$

where $I_{j}$ and $\kappa_{j}$ are the radiation intensity and the absorption coefficient of the jth gas, respectively. The term $a_{j}$ is denominated the temperature coefficient and represents the fraction of blackbody radiation emitted at the local temperature of the medium in the wavenumber interval correspondent to gas $j$; its dependence on the temperature $T$ is described by a polynomial function:

$$
\mathrm{a}_{\mathrm{j}}=\sum_{\mathrm{k}=0}^{4} \mathrm{~b}_{\mathrm{j}, \mathrm{k}} \mathrm{T}^{\mathrm{k}}
$$

The polynomial coefficients $b_{j, k}$ of this equation, as well as the value of the pressure-absorption coefficient $\kappa_{\mathrm{p}, \mathrm{j}}$ of each gray gas (defined as the ratio between the absorption coefficient and the partial pressure of the participating species), are obtained from fitting global radiation data. In this study, the coefficients presented by Dorigon et al. (2013) are adopted, obtained considering a mixture where water vapor and carbon dioxide are the only participating species and the ratio between their partial pressures is equal to 2 .

For the solution of the radiative heat transfer problem, Eq. (3) is solved $\mathrm{N}_{\mathrm{j}}+1$ times, where $\mathrm{j}=0$ denotes the transparent windows. The total radiation intensity is obtained as a summation of the intensity $I_{j}$ over all gray gases and transparent windows. To determine the temperature coefficient for the transparent windows, $\mathrm{a}_{0}$, the constraint $\sum_{j=0}^{N_{j}} a_{j}=1$, derived from the conservation of energy principle, is used.

\section{Numerical Procedures}

The set of equations described in the previous sections are numerically solved using the open-source 
,Fortran-based computer fluid dynamics code Fire Dynamics Simulator. The core algorithm of this solver consists in an explicit predictor-corrector scheme, with second order accuracy both in time and in space (McGrattan et al., 2017a).

The computational domain is discretized utilizing staggered, structured, rectilinear meshes. For the spatial discretization, an uniformly spaced grid with approximately 185000 volumes is used, with an aspect ratio of 3 between the dimensions of the cells in the $\mathrm{x}$-direction and in the $\mathrm{y}$ - and $\mathrm{z}$-directions. The time-step of the transient simulations is adjusted based on the Courant-Friedrichs-Lewy criterion, modified as to account for constraints of mass density and fluid volume realizability (McGrattan et al., 2017b) and kept below unity throughout all computations. The duration of each simulation, as well as the time interval used to compute the timeaverages of all quantities, are dependent on the inlet velocity of the fuel and will be discussed later. For the solution of the RTE with the finite volume method, 100 finite angles are used.

\section{RESULTS}

Turbulence-radiation interaction effects are analyzed by comparing time-averaged radiative quantities (namely, the radiative heat flux at the walls and the volumetric radiative heat source) obtained from transient LES computations with quantities determined from independent one time-step calculations initialized with the time-averaged temperature and species concentration fields (which are themselves calculated from data of the transient simulations). In this methodology, it is assumed that the former set of results include the effects of turbulence fluctuations (and, as a consequence, account for turbulence-radiation interaction), thus they are referenced in this paper as "with TRI" results, while the latter fully neglect those effects, being denominated "without TRI” results.

This methodology is applied to five test cases, all based on the problem described earlier. The only differences between the cases are the inlet velocity of the methane stream and the total duration of the transient simulations. The inlet velocity is defined as to provide Reynolds numbers Re (computed based on methane properties and on the diameter of the circular opening through which the fuel is injected) ranging from 5000 to 15000 in an arithmetic progression, which results in inlet velocities varying between $1 \mathrm{~m} / \mathrm{s}$ and $3 \mathrm{~m} / \mathrm{s}$. The duration of each transient simulation is defined as to allow an undisturbed methane stream to pass through the length of the domain thirty times, while averages of all quantities of interest are calculated considering approximately only the last two thirds of the total simulation time (the initial third of the duration of the simulations-i.e., the first ten passages of the undisturbed methane stream-is assumed to be the time necessary for the problem to reach a statistically steady condition). For example, for the case with $R e$ $=5000$, corresponding to an inlet velocity of the methane stream equal to $1 \mathrm{~m} / \mathrm{s}$, the problem is simulated for a total of $60 \mathrm{~s}$ and averages of all quantities are extracted from data corresponding to the final $40 \mathrm{~s}$ of simulation.

As a reference for the discussion that follows, Fig. 2 shows the time-averaged fields of temperature $\overline{\mathrm{T}}$ and radiative heat source $\overline{\mathrm{S}_{\mathrm{r}}}$ computed from the transient LES calculations for all cases considered. From this figure, the flame sheet is clearly identifiable by the region with peak temperatures and lower values of the radiative heat source (i.e., greater radiative emission). It should be noted that the fields of $\overline{\mathrm{S}}_{\mathrm{r}}$ shown in the figure correspond to the results obtained considering turbulence fluctuations, or the "with TRI” results.

Figure 3 depicts the distribution of mean radiative heat flux to the domain walls $\overline{\mathrm{q}}_{\mathrm{r}}$ along the
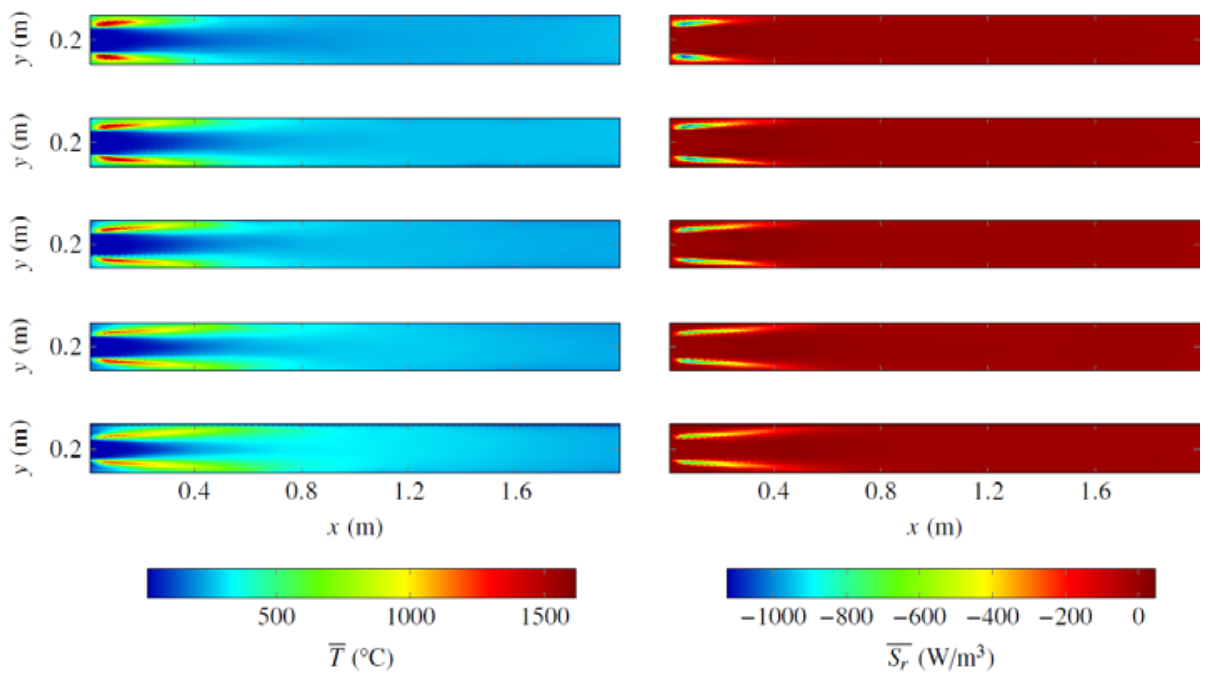

Figure 2. Time-averaged fields of temperature (left) and radiative heat source (right) along the mean longitudinal plane of the domain. From top to bottom: $R e=15$ 000, 12 500, 10 000, 7500 and 5000. 
longitudinal length of the domain, computed by the solutions considering and neglecting TRI effects (labeled "TRI" and "nTRI" in the figure, respectively). When TRI is neglected, a reduction is $\overline{\mathrm{q}}_{\mathrm{r}}$ is observed for all cases, especially in the first quarter of the domain, which corresponds to the flame region. A possible explanation for the largest deviations between "with TRI" and "without TRI" mean radiative heat fluxes being found near the flame region is that, at the flame front, elevated turbulent fluctuations of both temperature and species concentration are expected, which would increase the local magnitude of TRI effects. The same behavior is also noted for the mean total heat flux $\bar{q}_{t}$, which accounts both for radiative and convective heat transfer, as shown in Fig. 4.

A quantitative comparison between "with TRI" and "without TRI" results can be made by defining a normalized difference that, for an arbitrary mean quantity $\bar{\phi}$, may be written as:

$$
\psi_{\phi}=\left|\frac{\bar{\phi}^{\mathrm{TRI}}-\bar{\phi}^{\mathrm{nTRI}}}{\max \left(\bar{\phi}^{\mathrm{TRI}}\right)}\right|
$$

where $\bar{\phi}^{\text {TRI }}$ and $\bar{\phi}^{\text {nTRI }}$ are the values of $\bar{\phi}$ computed by solutions considering and neglecting TRI effects, respectively, and max ( $\phi^{\mathrm{TRI}}$ ) is the maximum value of $\phi^{\mathrm{TRI}}$ over the entire domain for a given case.

Following this definition, Fig. 5 shows the fields for the normalized difference between the mean radiative heat source computed considering and neglecting turbulence-radiation interaction, $\psi_{\mathrm{S}_{\mathrm{r}}}$, along the central longitudinal plane of the domain for all cases considered in this paper. It is possible to note that TRI effects, whose magnitude may be assessed by the local value of $\psi_{\mathrm{S}_{\mathrm{r}}}$, are more pronounced at the flame region, coinciding with the locations of peak temperature and peak radiative emission in Fig. 2, which indicates that the impact of turbulenceradiation interaction is greater precisely in the most important regions for the overall radiative heat transfer problem. This is corroborated by the fact that the largest differences between "with TRI" and "without TRI" wall radiative and total heat fluxes are also located closer to the flame, as discussed previously. Similar findings are also reported by other works on the subject (Coelho, 2007).

A more comprehensive and quantitative view on the magnitude of turbulence-radiation interaction is given by Tab. 1, which shows, for all cases considered in this paper, the maximum and the domain-averaged values of the normalized difference between "with TRI" and "without TRI" results for the mean radiative heat source and for the mean heat fluxes at the domain walls (radiative, convective and total). Following the nomenclature introduced in Eq. 5 , the normalized differences for the mean wall radiative, convective and total heat fluxes are referred to as $\psi_{\mathrm{q}_{\mathrm{r}}}, \psi_{\mathrm{q}_{\mathrm{c}}}$, and $\psi_{\mathrm{q}_{\mathrm{t}}}$, respectively.

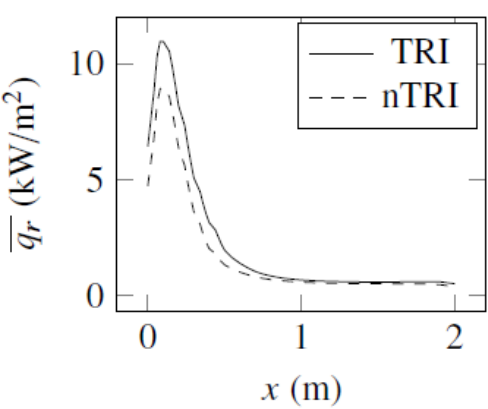

(a)

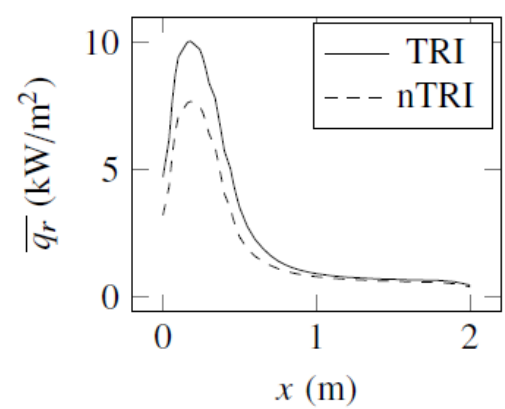

(b)

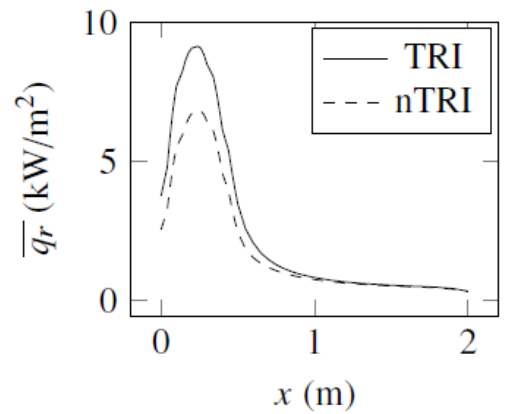

(c)

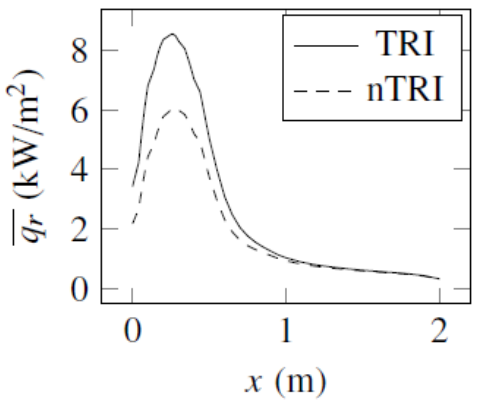

(d)

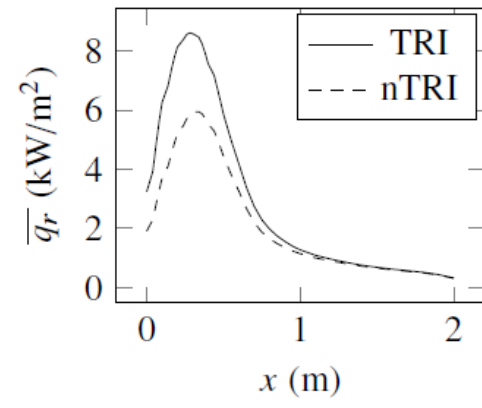

(e)

Figure 3. Wall radiative heat flux along the longitudinal direction of the domain: (a) $\operatorname{Re}=15$ 000; (b) $\operatorname{Re}=12$ 500; (c) $\operatorname{Re}=10$ 000; (d) $\operatorname{Re}=7500 ;$ (e) $\operatorname{Re}=5000$. 


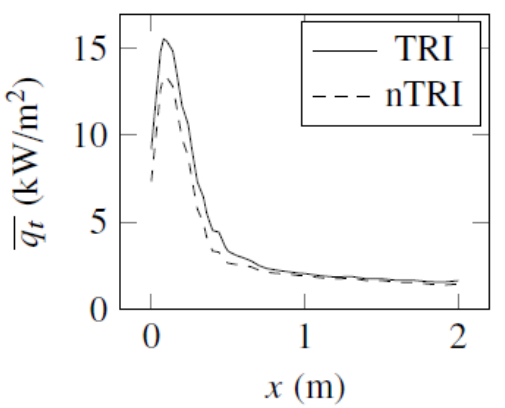

(a)

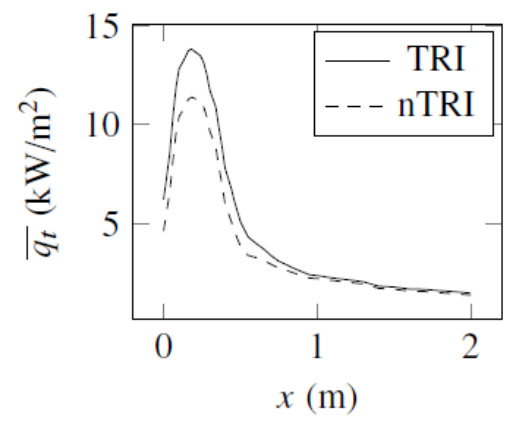

(b)

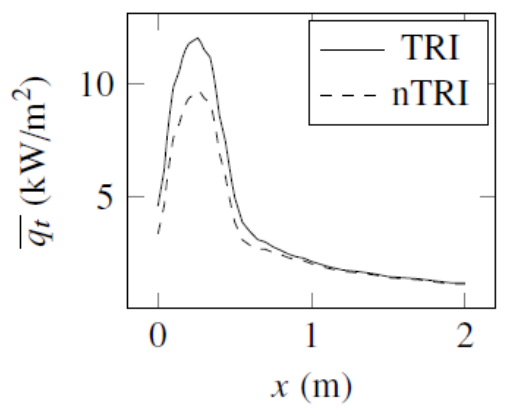

(c)

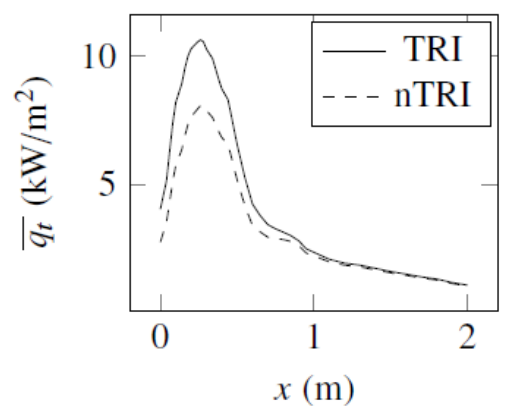

(d)

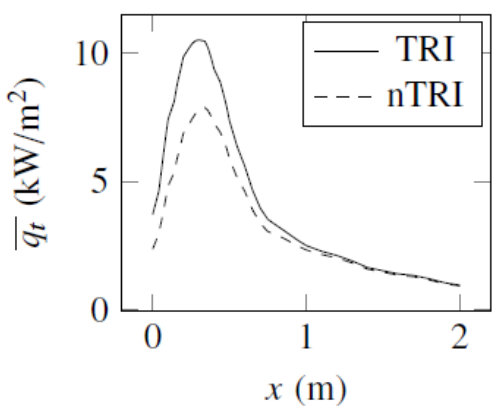

(e)

Figure 4. Wall total heat flux along the longitudinal direction of the domain: (a) $\operatorname{Re}=15$ 000; (b) $\operatorname{Re}=12$ 500; (c) $\operatorname{Re}=10$ 000; (d) $\operatorname{Re}=7500 ;$ (e) $\operatorname{Re}=5000$.

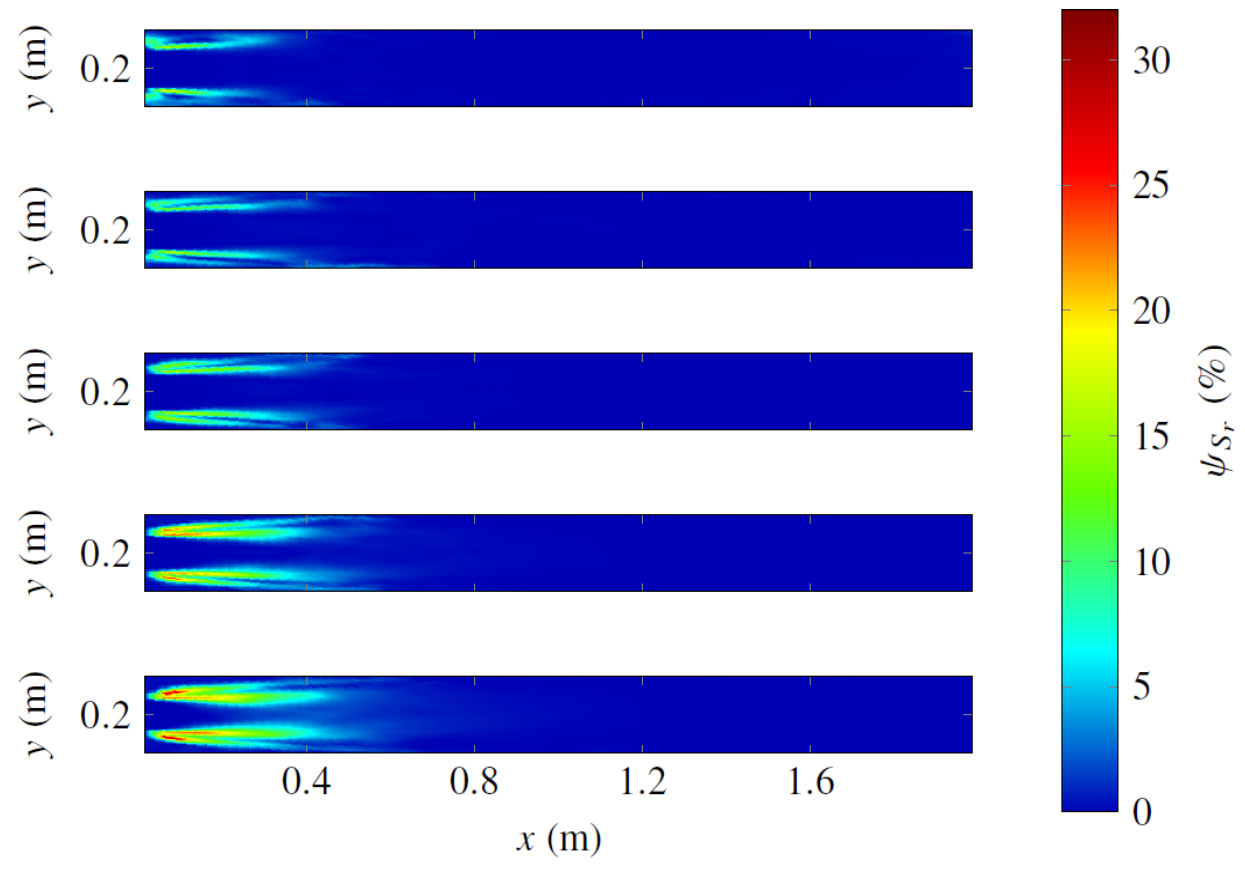

Figure 5. Normalized difference between the mean radiative heat source computed considering and neglecting TRI along the central longitudinal plane of the domain: (a) $\operatorname{Re}=150$ 000; (b) $\operatorname{Re}=12$ 500; (c) $\operatorname{Re}=10$ 000; (d) $\operatorname{Re}=7500$; (e) $\operatorname{Re}=5000$.

When measured by the peak normalized difference for both the mean radiative heat source and the mean wall radiative heat flux, TRI is responsible for a difference from 18 to $34 \%$ between results obtained including and neglecting the effects of turbulent fluctuations. This is not an insignificant contribution, indicating that the turbulence-radiation interaction phenomenon is important for the cases simulated in this paper, and is within the range of deviations reported by previous works on TRI in 
Table 1. Normalized difference between "with TRI" and "without TRI” results for a number of quantities.

\begin{tabular}{|c|c|c|c|c|c|c|c|c|}
\hline \multirow[b]{2}{*}{$\operatorname{Re}$} & \multicolumn{2}{|c|}{$\psi_{q_{r}}(\%)$} & \multicolumn{2}{|c|}{$\psi_{q_{c}}(\%)$} & \multicolumn{2}{|c|}{$\psi_{q_{t}}(\%)$} & \multicolumn{2}{|c|}{$\psi_{S_{r}}(\%)$} \\
\hline & Maximum & Average & Maximum & Average & Maximum & Average & Maximum & Average \\
\hline 5000 & 33.97 & 15.57 & 4.36 & 2.81 & 28.42 & 13.28 & 31.92 & 1.16 \\
\hline 7500 & 30.38 & 12.64 & 3.86 & 2.36 & 24.93 & 10.66 & 26.51 & 0.95 \\
\hline 10000 & 25.87 & 12.05 & 3.19 & 1.80 & 20.15 & 9.60 & 25.88 & 0.71 \\
\hline 12500 & 22.32 & 8.57 & 3.00 & 1.51 & 16.69 & 6.64 & 24.77 & 0.67 \\
\hline 15000 & 18.78 & 8.59 & 3.42 & 1.48 & 14.00 & 6.51 & 25.35 & 0.54 \\
\hline
\end{tabular}

methane-air diffusion flames (Jeng et al., 1984; Mazumder and Modest, 1999; Li and Modest, 2002). Averaged values of $\psi_{\mathrm{S}_{\mathrm{r}}}$ and $\psi_{\underline{a}_{\mathrm{r}}}$ given in Tab. 1 are lower because they are averages of the local difference over the entire domain (or the entire surface area of the domain walls) and, as a consequence, are influenced by the smaller $\psi$ outside the flame region.

The importance of TRI effects to the mean total heat flux, although not negligible, is not as significant (this may also be noted by comparing Figs. 3 and 4). An explanation for this lies in the smaller influence of turbulence-radiation interaction on the mean convective heat flux $\psi_{\mathrm{q}_{c}}$, which tends to dampen the overall impact of TRI on $\psi_{\mathrm{q}_{\mathrm{t}}}$. As indicated by Tab. 1, the difference between "with TRI" and "without TRI" results for the mean convective heat flux at the domain walls is, at most, approximately $4 \%$. A small contribution of TRI to $\psi_{\mathrm{q}_{\mathrm{c}}}$ is expected, since convective heat transfer is an almost totally linear process and turbulence-radiation interaction is promoted by non-linearities between the governing equations (Coelho, 2007).

A curious finding, indicated both by Fig. 5 and Tab. 1, is that, as the Reynolds number of the inlet fuel stream increases, the importance of TRI effects decrease. This is counter-intuitive, since an increase in the Reynolds number tends to also increase the magnitude of turbulent fluctuations, which should contribute to widen the difference between "with TRI" and "without TRI" results. However, such explanation apparently does not hold true in practice, as already noted by other authors-for example, Mazumder and Modest (1999) and Modest (2005), both analyzing methane-air diffusion flames, report that changing the Reynolds number did not appreciably alter the difference between results considering and neglecting TRI effects.

Before concluding, an observation on the accuracy of the numerical results reported in this paper must be made. The present study consists in an initial investigation on turbulence-radiation interaction in reactive problems, and, as such, its main focus is the development of the methodology of analysis-which includes the creation and implementation of in-house subroutines to the FDS base-code, the generation and post-processing of the transient LES data and the definition of an approach to evaluate the differences between "with TRI" and "without TRI" results. Therefore, details of the numerical simulations were not investigated in-depth: for example, the spacing of the grid was not optimized, the quality of the spatial and angular discretizations was not rigorously evaluated, and the boundary conditions adopted could be improved (specially the thermal boundary condition at the open surface corresponding to the domain's outlet, where a constant temperature equal to the ambient temperature was prescribed). As a consequence, results such as the mean temperature and the mean radiative heat source fields depicted in Fig. 2 may not be as accurate as possible. However, since TRI was evaluated as a comparison between "with TRI" and "without TRI" quantities that were calculated by the same mesh and with the same boundary conditions, one should expect that the normalized differences obtained in this paper are representative of the studied problem.

\section{CONCLUSIONS}

This study presented an analysis on turbulenceradiation interaction in a methane-air diffusion flame. In order to assess the magnitude of TRI effects, mean quantities obtained from transient computations using Large Eddy Simulation were compared to the quantities determined from independent one-time step calculations initialized using mean temperature and species concentration fields. The finite volume method for the spatial integration and the WeightedSum-of-Gray-Gases spectral model were adopted to solve the radiative heat transfer equation, and all simulations were conducted in the solver Fire Dynamics Simulator.

Five test cases were tested, with the Reynolds numbers of the fuel stream ranging from 5000 to 15000. Turbulence-radiation interaction was found to be responsible for differences between predicted mean radiative quantities considering and neglecting turbulent fluctuations as high as $34 \%$. Locally, the largest deviations were observed at the flame region, probably because of to the elevated fluctuations of temperature and species concentrations that occur in that region. Compared to the mean radiative heat flux, TRI effects were smaller for the mean total heat flux and negligible for the mean convective heat flux, due to the almost linear characteristic of convective heat transfer. As the Reynolds number increased, the magnitude of the turbulence-radiation interaction effects showed a slight decrease, in an apparently counterintuitive result; however, previous studies on 
methane-air diffusion flames report that it is not expected a significant influence of the Reynolds number on the importance of TRI effects to the problem.

\section{ACKNOWLEDGEMENTS}

This work was supported by a doctoral scholarship by CNPq.

\section{REFERENCES}

Cassol, F., Brittes, R., França, F. H. R., and Ezekoye, O. A., 2014, Application of the WeightedSum-of-Gray-Gases Model for Media Composed of Arbitrary Concentrations of $\mathrm{H} 2 \mathrm{O}, \mathrm{CO} 2$ and Soot, International Journal of Heat and Mass Transfer, Vol. 79, pp. 796-806.

Coelho, P. J., 2007, Numerical Simulation of the Interaction between Turbulence and Radiation in Reactive Flows, Progress in Energy and Combustion Science, Vol. 33, pp. 311-383.

Dorigon, L. J., Duciak, G., Brittes, R., Cassol, F., Galarça, M., and França, F. H. R., 2013, WSGG Correlations based on HITEMP2010 for Computation of Thermal Radiation in Non-Isothermal, NonHomogeneous H2O/CO2 Mixtures, International Journal of Heat and Mass Transfer, Vol. 64, pp. 863873.

Germano, M., Piomelli, U., Moin, P., and Cabot, W. H., 1991, A Dynamic Subgrid-Scale Eddy Viscosity Model, Physics of Fluids A, Vol. 64, No. 7, pp. 1760-1765.

Howell, J. R., Siegel, R., and Mengüç, M. P., 2010, Thermal Radiation Heat Transfer, CRC Press, 5th edition, United States of America.

Jarrin, N., 2008, Synthetic Inflow Boundary Conditions for the Numerical Simulation of Turbulence, Ph.D. Thesis, University of Manchester, Manchester, United Kingdom.

Jeng, S., Lai, M., and Faeth, G. M., 1984, Nonluminous Radiation in Turbulent Buoyant Axisymmetric Flames, Combustion Science and Technology, Vol. 4, No. 1-4, pp. 41-53.

Li, G., and Modest, M. F., 2002, Application of Composition PDF Methods in the Investigation of Turbulence-Radiation Interactions, Journal of Quantitative Spectroscopy and Radiative Transfer, Vol. 73, No. 2-5, pp. 461-472.

Lilly, D. K., 1992, A Proposed Modification of the Germano Subgrid-Scale Closure Method, Physics of Fluids A, Vol. 4, No. 3, pp. 633-635.

Mazumder, S., and Modest, M. F., 1999, A Probability Density Function Approach to Modeling Turbulence-Radiation Interactions in Nonluminous Flames, International Journal of Heat and Mass Transfer, Vol. 42, pp. 971-991.

McGrattan, K., Hostikka, S., McDermott, R., Floyd, J.,Weinschenk, C., and Overholt, K., 2017a, Fire Dynamics Simulator User's Guide, NIST
Special Publication, 1019, 6th edition, Baltimore, Maryland.

McGrattan, K., Hostikka, S., McDermott, R., Floyd, J.,Weinschenk, C., and Overholt, K., 2017b, Fire Dynamics Simulator Technical Reference Guide Volume 1: Mathematical Model. NIST Special Publication, 1018-1, 6th edition, Baltimore, Maryland.

Modest, M. F., 2005, Multiscale Modeling of Turbulence, Radiation and Combustion Interactions in Turbulent Flames, International Journal for Multiscale Computational Engineering, Vol. 3, pp. 85-105.

Modest, M. F., 2013, Radiative Heat Transfer, Academic Press, 3rd edition, United States of America.

Poinsot, T., and Veynante, D., 2005, Theoretical and Numerical Combustion, RT Edwards, Inc., 2nd edition, Philadelphia, Pennsylvania.

Raithby, G. D., and Chui, E. H., 1990, A FiniteVolume Method for Predicting Radiant Heat Transfer in Enclosures with Participating Media, Journal of Heat Transfer, Vol. 112, No. 2, pp. 415-423.

Rehm, R. G., and Baum, H. R., 1978, The Equations of Motion for Thermally Driven, Buoyant Flows, Journal of Research of the National Bureau of Standards, Vol. 83, No. 3, pp. 297-308. 